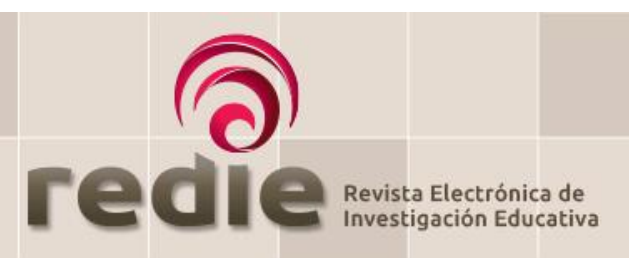

Vol. 21, 2019/e26

\title{
Compromiso organizacional y promedio escolar en estudiantes de maestría: el efecto moderador de las tendencias alocéntricas e idiocéntricas
}

\author{
Organizational Commitment and Grade Point Average in MBA \\ Students: The Moderating Effect of Allocentric and Idiocentric \\ Tendencies
}

Daniel Arturo Cernas Ortiz (*) dacernaso@uaemex.mx

Rosa María Nava Rogel (*) rosanr06@yahoo.com.mx

(*) Universidad Autónoma del Estado de México

(Recibido: 4 de agosto de 2017; Aceptado para su publicación: 3 de enero de 2018)

Cómo citar: Cernas. D. A. y Nava, R. M. (2019). Compromiso organizacional y promedio escolar en estudiantes de maestría: el efecto moderador de las tendencias alocéntricas e idiocéntricas. Revista Electrónica de Investigación Educativa, 21, e26, 1-10. doi:10.24320/redie.2019.21.e26.2122

\section{Resumen}

El objetivo de este trabajo fue examinar la relación del compromiso organizacional con el promedio escolar en estudiantes de la Maestría en Administración de la Universidad Autónoma del Estado de México. También se analizó si las tendencias alocéntricas e idiocéntricas de los alumnos moderan esta relación. Se aplicó una encuesta en la universidad mencionada $(n=80)$. Los resultados indican que el compromiso organizacional y el promedio escolar tienen una relación negativa que se amplifica cuando las tendencias alocéntricas son altas y que se reduce cuando las altas son las tendencias idiocéntricas. En general, el compromiso organizacional es deseable para las organizaciones pero podría tener efectos perniciosos en las calificaciones de las personas que estudian y trabajan.

Palabras clave: Promedio de calificaciones, educación y trabajo, posgrado, administración de empresas.

\section{Abstract}

The aim of this study was to examine the relationship between organizational commitment and grade point average (GPA) in MBA students. We also analyzed whether the students' allocentric and idiocentric tendencies moderate this relationship. A survey was carried out in the university in mention $(n=80)$. The results indicate that organizational commitment and GPA have a negative relationship that amplifies at high levels of allocentric tendencies and diminishes when idiocentric tendencies are high. In general, organizational commitment is desirable for organizations but it could have negative effects on the grades of those persons who simultaneously study and work. 


\section{Introducción}

A nivel maestría muchos estudiantes combinan la escuela con el trabajo, sin embargo esta combinación puede ser problemática, a tal grado que el Consejo Nacional de Ciencia y Tecnología de México otorga becas para que algunos estudiantes dediquen tiempo completo a sus estudios (Conacyt, 2017). En estudiantes de maestría, por lo tanto, el entorno laboral es un factor para entender por qué algunos alumnos tienen un mejor nivel de aprovechamiento que otros.

El promedio escolar es un indicador clave del aprovechamiento estudiantil y refleja no sólo factores de carácter individual como la inteligencia (Coyle, 2015), el compromiso con los estudios (Alshehri, 2017) y la personalidad (Vedel, Thomsen y Larsen, 2015), sino también aspectos sociales como el ambiente familiar (Ong, Phinney y Dennis, 2006) y, en estudiantes que trabajan, el entorno laboral (Rochford, Connolly y Drennan, 2009). Con respecto al trabajo, algunas posturas indican que éste tiene un efecto positivo en el desempeño estudiantil, pues mejora las habilidades para cumplir con las expectativas de supervisores (y de los docentes), administrar el presupuesto personal y ser puntual (Philips y Sandstrom, 1990). Otros estudios argumentan que trabajar afecta las calificaciones, ya que eleva la presión de tiempo y las dificultades para cumplir con las demandas escolares y los compromisos familiares, además de que drena la energía y la atención (Marsh, 1991). La evidencia apoya ambos lados del debate (Patton y Smith, 2009), de tal suerte que se requiere investigar a fondo los fenómenos laborales que impactan el desempeño escolar.

El Compromiso Organizacional (co) es un elemento laboral que puede influenciar el aprovechamiento escolar. El co es la fuerza relativa de la identificación personal y el involucramiento en una organización en particular (Mowday, Porter y Steers, 1982). Este factor es clave para entender los retardos y la permanencia (Meyer, Stanley, Herscovitch y Topolnytsky, 2001) y aun comportamientos como el de ciudadanía (Ng y Feldman, 2011) y el innovador (Vinarski-Peretz, Binyamin y Carmeli, 2011). En consecuencia, las organizaciones están interesadas en mantener una fuerza de trabajo comprometida. Sin embargo, un alto co podría ser un arma de doble filo para el empleado que estudia una maestría. Por un lado, un fuerte compromiso se asocia a un alto desempeño laboral y a recompensas (Meyer et al., 2001), y por otro, se relaciona con una alta inversión de tiempo y esfuerzo en asuntos laborales (Mowday et al., 1982). Puesto que el tiempo y la energía de las personas son finitas, una inversión considerable de tiempo en asuntos de la organización podría impactar perniciosamente al promedio (Carrillo y Ríos, 2013).

Como se infiere de lo anterior, en los alumnos de maestría que trabajan el co puede ser un factor que afecte el tiempo disponible para los estudios, la atención, la energía y, en consecuencia, el desempeño académico. En este sentido, un objetivo de este trabajo es examinar si el co de una muestra de 80 estudiantes de Maestría en Administración de la Universidad Autónoma del Estado de México tiene una relación negativa con su promedio escolar. El programa en mención se ha ofertado por más de 40 años y es la primera opción para cursar una Maestría en Administración en una institución pública del Estado de México (ANUIES, 2016).

El fenómeno descrito resulta más intrigante si se considera que las tendencias alocéntricas e ideocéntricas de los alumnos pueden amplificar (o disminuir) los efectos del co en sus calificaciones. Mientras que el alocentrismo se refiere a la propensión individual a enfatizar la importancia de las relaciones sociales y el mantenimiento de la interdependencia, el idiocentrismo es la propensión a enfatizar la libertad personal, la autoexpresión y la independencia (Miller, 1988). Las tendencias alocéntricas e idiocéntricas influyen en el grado en que las personas dan prioridad a las metas de un grupo (por ejemplo las de la organización) o a las propias (las escolares) (Triandis, Leung, Villareal y Clack, 1985; Yamaguchi, Kuhlman y Sugimori, 1995). Por lo tanto, la relación entre el co y el promedio escolar podría variar de acuerdo a la prevalencia de tales tendencias en los alumnos de maestría. Con base en estos antecedentes, el segundo objetivo de este trabajo es analizar el papel moderador de las tendencias mencionadas en la posible relación negativa entre el co y el promedio escolar.

Con base en la teoría del intercambio social, el co es una actitud favorable del empleado que emerge en reciprocidad al trato que recibe de la organización (Cropanzano y Mitchell, 2005). Por ejemplo, un 
empleado experimenta un alto compromiso cuando percibe que la organización valora sus contribuciones, se preocupa por su bienestar y satisface sus necesidades socioemocionales (Rhoades y Eisenberger, 2002). Cuando el compromiso germina, el empleado "se une" a la organización esforzándose por realizar acciones beneficiosas para ella (por ejemplo, no faltar y hacer mejor su trabajo) (Meyer, Becker y Van Dick, 2006). Incluso, los empleados altamente comprometidos realizan tareas extra a sus labores, como ayudar a otros a cumplir sus objetivos ( $\mathrm{Ng}$ y Feldman, 2011). Un alto co, por lo tanto, implica una alta inversión de tiempo, esfuerzo y atención en asuntos de la organización.

Si bien el tiempo dedicado al trabajo es importante, el dedicado a los estudios es crítico para el aprovechamiento académico. La experiencia indica que cuando los alumnos dedican tiempo a sus labores escolares (llegar a tiempo a clases, por ejemplo) tienden a sacar mejores calificaciones. Sin embargo, el efecto que el tiempo dedicado a los estudios tiene en el promedio escolar no es del todo claro en la literatura. Algunas evidencias sugieren que, en detrimento de los estudios, el tiempo laboral tiene un efecto negativo en las calificaciones después de las primeras 20 horas de trabajo semanal (Mortimer, Finch, Seongryeol, Shanahan y Call, 1996). Otras evidencias sugieren que el tiempo dedicado a los estudios podría no tener un efecto directo en las calificaciones, sino que podría interactuar con la habilidad intelectual para afectar el desempeño (Nonis y Hudson, 2006). Al margen de si el tiempo dedicado a los estudios tiene un efecto directo o interactivo en el promedio, en una muestra de alumnos de licenciatura (pregrado) de la Universidad de Guadalajara (México), Carrillo y Ríos (2013) encontraron que si los estudiantes aumentan una hora su jornada laboral, el efecto negativo sobre su promedio podría ser de entre - 0.850 y -0.232 puntos. Con base en estos antecedentes se postula la hipótesis 1: el co tiene una relación negativa con el promedio escolar de los estudiantes de Maestría en Administración.

Sin embargo, los efectos negativos del co en el promedio escolar se pueden amplificar cuando el alocentrismo es elevado. El alocentrismo se relaciona con la armonía social, la modestia (restar importancia a los logros individuales, por ejemplo), una alta sensibilidad al rechazo social y una alta necesidad de afiliación (Caldwell-Harris, 2006). Estas características, por supuesto, no implican que las personas predominantemente alocéntricas no se pongan metas ambiciosas (Oettingen, 1995) sino que tienden a ponerse objetivos acordes a los de sus grupos sociales relevantes (Triandis et al., 1985) y cuando sus metas y las del grupo difieren, las del grupo son prioridad (Yamaguchi et al., 1995). Como se infiere, cuando el alocentrismo es alto los empleados comprometidos podrían dar más prioridad a cumplir con las metas de la organización (un grupo social muy relevante para ellos) que a las personales (maximizar su desempeño en la maestría). En consecuencia, en condiciones de un alto alocentrismo, el tiempo, la energía y la atención de las personas comprometidas con su organización se dedicarán más a actividades relacionadas con el trabajo que a aquellas relacionadas con la maestría. Formalmente, se postula la hipótesis 2: las tendencias alocéntricas moderan la relación negativa entre el co y el promedio escolar de los estudiantes de Maestría en Administración en una forma tal que la relación es más negativa cuando el alocentrismo es alto.

Por el contrario, los efectos negativos del co en el promedio podrían ser menores cuando el idiocentrismo es elevado. El idiocentrismo se caracteriza por la necesidad de reconocimiento social, la competencia y las aspiraciones de poder (Caldwell-Harris, 2006); las personas con predominancia idiocéntrica no sólo trabajan mejor solas, sino también cuando tienen injerencia en la decisión de qué, cómo y cuándo hacer las cosas (Triandis, 2001). El idiocentrismo, en consecuencia, resalta la necesidad de fijarse metas independientemente de los grupos sociales relevantes para los individuos (Triandis et al., 1985) y de dar prioridad a las metas propias cuando éstas y las del grupo discrepan (Yamaguchi et al., 1995). Como se implica en este caso, cuando el idiocentrismo es alto -por muy comprometidos que los alumnos estén con su organización- se decantarán por maximizar su desempeño escolar. Por consiguiente, cuando el idiocentrismo es elevado, el tiempo, la energía y la atención se dedicarían un poco menos a las labores relacionadas a la organización para maximizar el promedio escolar. En consecuencia, se propone la hipótesis 3: las tendencias idiocéntricas moderan la relación negativa entre el co y el promedio escolar de los estudiantes de Maestría en Administración en una forma tal que la relación es menos negativa cuando el idiocentrismo es alto. 


\section{Método}

Este estudio es cuantitativo, no experimental, transversal y correlacional debido a que las variables involucradas no se cuantifican en un ambiente controlado para observar causas y efectos, se miden en un único momento en el tiempo y se correlacionan para observar asociaciones en términos probabilísticos.

Durante el semestre de primavera de 2017 estaban inscritos 118 alumnos en la Maestría en Administración de la Universidad Autónoma del Estado de México, para el estudio se contó con la participación de 85 estudiantes (muestra no probabilística del $72 \%$ del total de la matrícula). Después de eliminar las respuestas incompletas, la base de datos final se compuso de 80 casos (47 mujeres y 33 hombres). El promedio de edad fue de 30.26 años con una desviación estándar de 4.67; los estudiantes considerados tenían empleo al momento de la recolección de datos y la mayoría reportó un ingreso mensual neto de entre 6,000 y 20,000 pesos mexicanos (61.71\%).

Los datos se recolectaron mediante una encuesta auto-administrada, el cuestionario se entregó a los profesores para que lo aplicaran durante las horas de clase. En el instrumento no se pidieron datos de identificación (nombre del alumno) ni se comunicaron los objetivos de la investigación. Estas acciones evitaron, en la medida de lo posible, sesgos que podrían contaminar las respuestas individuales (como deseabilidad social, condescendencia y correlaciones ilusorias).

\subsection{Operacionalización de variables}

Promedio escolar. Se midió como el promedio simple autorreportado de las calificaciones que los alumnos tenían al momento de la aplicación de la encuesta.

Compromiso Organizacional. Se midió por medio de la escala de ocho reactivos de compromiso organizacional afectivo de Allen y Meyer (1990) $(a=0.87)$. Los reactivos se presentaron en un formato tipo Likert de siete puntos (donde 1=muy en desacuerdo y 7=muy de acuerdo). La oración "Yo estaría muy feliz de pasar el resto de mi carrera en esta organización" ejemplifica la escala. Sólo se midió la dimensión afectiva del modelo de Allen y Mayer (1990) debido a las fuertes dudas sobre si las dimensiones normativa y de continuidad representan una actitud genuina hacia la organización o pensamientos de obligación y de falta de oportunidades (Solinger, van Olffen y Roe, 2008). La equiparación del compromiso afectivo al compromiso organizacional per se es común en la investigación sobre el tema (Vinarski-Peretz et al., 2011).

Tendencias alocéntricas. Se empleó la escala de colectivismo horizontal de Singelis, Triandis, Bhawuk y Gelfand (1995) en su versión corta, como se presenta en Triandis y Gelfand (1998) (a=0.68). Los cuatro reactivos de la escala se presentaron en un formato tipo Likert de nueve graduaciones (donde 1=nunca y 9=siempre). La oración "El bienestar de mis colegas es importante para mí" ejemplifica la escala.

Nótese que la escala de Singelis et al. (1995) se denomina colectivismo debido a una diferencia de nivel de análisis. La escala mide alocentrismo cuando se aplica para estimar diferencias individuales en tendencias colectivistas. La escala mide colectivismo cuando los datos individuales se agregan para estimar diferencias entre grupos. En términos conceptuales, el alocentrismo y el colectivismo son lo mismo, pero uno tiene significado a nivel individual y el otro a nivel social. La medición del alocentrismo por medio de escalas de colectivismo es común en la literatura (Caldwell-Harris, 2006).

Respecto a su confiabilidad, la escala raramente puede alcanzar el límite convencional de 0.70 sin que esto represente un problema grave. El concepto de colectivismo (alocentrismo) tiene un dominio muy amplio que es difícil capturar en unas cuantas preguntas, por lo que su medición es "más amplia que fiel" y arroja estimados de confiabilidad más bajos de lo habitual (Singelis et al., 1995). Además, el colectivismo es una dimensión cultural en la que se han detectado diferencias significativas entre los países (Hofstede, Hofstede y Minkvov, 2010).

Dado que los alumnos involucrados en el estudio pertenecen a la misma cultura (mexicana), sus 
respuestas a la escala tienden a parecerse. Una baja variación en las respuestas reduce la intercorrelación de los reactivos (Kerlinger y Lee, 2006) y, como resultado, también el Alpha de Cronbach.

Tendencias idiocéntricas. Se empleó la escala de individualismo horizontal de Singelis et al. (1995) en su versión corta, como la presentan Triandis y Gelfand (1998) ( $a=0.67$ ). Los cuatro reactivos se presentaron en un formato tipo Likert de nueve graduaciones (donde 1=nunca y 9=siempre). La oración "Mi identidad personal, independiente de otros, es muy importante para mí" ejemplifica esta medición. En este caso también aplica la discusión sobre el nombre de la escala de acuerdo al nivel de análisis (individualismo versus idiocentrismo) y el de la confiabilidad de la medida.

Antes de continuar, cabe destacar que todos somos tanto alocentristas como idiocentristas. En este trabajo ambas tendencias se midieron de forma simultánea en las mismas personas aunque, a menudo, una tendencia es más dominante.

Todas las escalas de medición fueron traducidas de su original en inglés al español (versión mexicana) a través de un proceso de traducción-retraducción. Primero, uno de los autores de este documento tradujo las escalas de su idioma original al español. Luego, un individuo ajeno al proyecto retradujo las preguntas al inglés. La versión retraducida se comparó con la original para detectar discrepancias y corregirlas. El proceso se repitió hasta que ambas versiones se consideraron equivalentes en términos gramaticales no necesariamente literales.

\section{Análisis y resultados}

\subsection{Descriptivos, confiabilidad y validez}

La tabla I muestra la media y la desviación estándar de los conceptos involucrados. Como se observa, el promedio estudiantil está arriba de 9 puntos. En cuanto al co, el nivel es mediano si se toma en cuenta que el tope máximo de la escala fue de 7. También se observa que el promedio de alocentrismo fue superior al de idiocentrismo, y esto es congruente con los hallazgos de Hofstede et al. (2010), que indican que la cultura mexicana es predominantemente colectivista. En la tabla también se muestra que la confiablidad del co presenta niveles aceptables (>0.7). Las alfas de alocentrismo e idiocentrismo están por debajo del valor convencional, pero dentro del margen esperado de acuerdo a lo discutido en párrafos anteriores. Las correlaciones son moderadas y destaca por su significatividad la negativa entre el promedio escolar y el co, y la positiva entre este factor y las tendencias alocéntricas. Aunque no se muestran, los valores de varianza extraída promedio (AVE) de los conceptos son cercanos a 0.5, lo que evidencia su validez (Hair, Black, Babin, Anderson y Tatham, 2005).

Tabla I. Estadística descriptiva y correlaciones bivariadas

\begin{tabular}{l|c|c|c|c|c|c}
\hline \multicolumn{1}{c|}{ Variables } & Media & $\begin{array}{c}\text { Desviación } \\
\text { estándar }\end{array}$ & a & 1 & 2 & 3 \\
\hline 1 Promedio escolar & 9.18 & 0.94 & & & & \\
2 Compromiso organizacional & 4.41 & 1.16 & 0.77 & $-0.24^{*}$ & & \\
3 Tendencias alocéntricas & 7.90 & 0.90 & 0.62 & -0.04 & $0.23^{*}$ & \\
4 Tendencias ideocéntricas & 7.51 & 1.17 & 0.60 & 0.18 & -0.02 & 0.18 \\
\hline
\end{tabular}

$*=p<0.05$

Fuente: Elaboración propia.

\subsection{Prueba de hipótesis}

Se utilizó el análisis de regresión simple y múltiple siguiendo las instrucciones de Aiken y West (1991) para examinar moderación. La prueba de moderación debe incluir en la misma ecuación el efecto principal de la variable independiente (compromiso), el efecto principal de la variable moderadora (tendencias alocéntricas) y la interacción entre la variable independiente y la moderadora que se forma por el producto de las dos. Si la interacción resulta significativa, la relación entre la variable independiente y la 
dependiente varía en fuerza (y quizás en dirección) a través de los valores de la variable moderadora. Se debe de tomar en cuenta el signo del coeficiente de la interacción para determinar si la relación bajo análisis se fortalece o se debilita. Como lo indican Aiken y West (1991), se utilizaron variables centradas a la media para evitar problemas de multicolinealidad.

La hipótesis 1 sugiere que el promedio escolar y el co se relacionan negativamente. En el Modelo 1 (ver tabla II) se observa que el coeficiente del co es negativo y significativo, lo que apoya esta hipótesis.

Tabla II. Coeficientes de regresión sobre el promedio escolar

\begin{tabular}{|c|c|c|c|}
\hline Variables & Modelo 1 & Modelo 2 & Modelo 3 \\
\hline Constante & $\begin{array}{c}0.00 \\
(0.11)^{\mathrm{a}}\end{array}$ & $\begin{array}{c}0.94 \\
(1.02)\end{array}$ & $\begin{array}{c}0.01 \\
(0.10)\end{array}$ \\
\hline Compromiso organizacional & $\begin{array}{c}-0.20^{*} \\
(0.09)\end{array}$ & $\begin{array}{c}-0.19^{*} \\
(0.09)\end{array}$ & $\begin{array}{l}-0.26^{* *} \\
(0.08)\end{array}$ \\
\hline Tendencias alocéntricas & & $\begin{array}{c}-0.11 \\
(0.13)\end{array}$ & \\
\hline Compromiso organizacional x tendendencias alocéntricas & & $\begin{array}{l}-0.21^{* *} \\
(0.08)\end{array}$ & \\
\hline Tendencias idiocéntricas & & & $\begin{array}{c}0.21^{*} \\
(0.09)\end{array}$ \\
\hline Compromiso organizacional x tendendencias idiocéntricas & & & $\begin{array}{l}0.30 * * * \\
(0.09)\end{array}$ \\
\hline $\mathrm{R}^{2}$ & $5.8 \%$ & $14.0 \%$ & $20.9 \%$ \\
\hline $\mathrm{R}^{2}$ ajustada & $4.5 \%$ & $10.5 \%$ & $17.7 \%$ \\
\hline $\mathrm{F}$ & $4.67 *$ & $4.01 * *$ & $6.52^{* * *}$ \\
\hline
\end{tabular}

La hipótesis 2 indica que las tendencias alocéntricas moderan la relación negativa entre el co y el promedio escolar en una forma tal que el efecto del compromiso en el promedio es más negativo cuando el alocentrismo es elevado. El Modelo 2 (ver tabla II) muestra que el coeficiente de la interacción es negativo y significativo e indica que, en efecto, la relación negativa entre el co y el promedio es más pronunciada cuando las tendencias alocéntricas son altas. Un análisis más a fondo respalda esta evidencia. Рara esto, la base de datos se dividió con referencia a la mediana de las puntuaciones de las tendencias alocéntricas. Debajo de la mediana quedaron 34 observaciones (bajas en tendencias alocéntricas) y arriba de ella 46 (altas en tendencias alocéntricas); en cada caso se regresó el promedio sobre el co en forma independiente. Los resultados indican que en los casos altos en tendencias alocéntricas el coeficiente del co es de $-0.30(p<0.05)$ y en los bajos de $-0.03(p>0.05)$. Este hallazgo indica que no sólo el efecto del co en el promedio es bastante más fuerte cuando el alocentrismo es alto, sino que cuando estas tendencias son bajas no hay relación significativa entre las variables. En conjunto, la evidencia apoya la hipótesis 2.

La hipótesis 3 predice que las tendencias idiocéntricas moderan la relación negativa entre el co y el promedio escolar de forma tal que el efecto del compromiso en el promedio es menos negativo cuando el idiocentrismo es elevado. El Modelo 3 (ver tabla II) indica que el coeficiente de la interacción es positivo y altamente significativo sugiriendo que la relación entre el co y el promedio escolar mengua cuando las tendencias idiocéntricas son altas. Para comprender mejor este resultado, una vez más se dividió la base de datos tomando como referencia la mediana de las puntuaciones de las tendencias idiocéntricas. Debajo de la mediana quedaron 37 observaciones (bajas en tendencias idiocéntricas) y arriba de ella 43 (altas en tendencias idiocéntricas). De forma independiente, en cada caso se regresó el promedio sobre el co. Los resultados muestran que en los casos altos en tendencias idiocéntricas el coeficiente del co es de $0.03(p>0.05)$, y en los bajos de $-0.46(p<0.01)$. Este hallazgo indica que cuando el idiocentrismo es alto el compromiso podría tener un pequeño efecto positivo en el promedio (aunque no se muestre significativo), y que cuando es bajo el efecto negativo del compromiso en el promedio se pronuncia considerablemente. De hecho, la $\mathrm{R}^{2}$ del Modelo 3 es la mayor de las reportadas en la tabla II. En su 
conjunto, la evidencia sugiere un fuerte apoyo para la hipótesis 3.

Como nota adicional, ninguno de los modelos de regresión presentó valores de inflación de varianza (VIF) mayores a 1.5; es decir, no hubo problemas de multicolinealidad.

\section{Discusión y conclusiones}

El co podría no ser benéfico para los empleados como estudiantes. Estudios previos indican que el tiempo dedicado al trabajo puede afectar de forma negativa las calificaciones (Carrillo y Ríos, 2013). Los resultados mostrados en este documento respaldan esta evidencia, pues si los empleados altamente comprometidos dedican más tiempo a sus labores (Ng y Feldman, 2011), a mayor co menor promedio. Sin embargo, los hallazgos reportados van más allá de respaldar indirectamente investigaciones previas. El co es un fenómeno que no sólo puede manifestarse en una mayor inversión de tiempo en asuntos laborales, sino que también implica una mayor atención y esfuerzo en asuntos de la organización (Meyer et al., 2001). De tal suerte, quizá no sea el tiempo per se invertido en el trabajo lo que impacta de forma negativa el promedio estudiantil, sino la atención y el esfuerzo que se dedica a los asuntos organizacionales; investigaciones posteriores deberían aclarar este asunto.

El presente documento contribuye también a entender mejor el papel de las diferencias individuales en el desempeño académico. En este trabajo se muestra que las diferencias individuales aprendidas tienen un efecto que amplifica o debilita la influencia de aspectos sociales (por ejemplo, el trabajo) en las calificaciones. Las tendencias alocéntricas e idiocéntricas representan diferencias en la propensión a priorizar las metas grupales sobre las individuales (o viceversa). Los hallazgos de este estudio sugieren que el efecto negativo del compromiso es más probable cuando el alocentrismo es alto, pero sobre todo cuando el idiocentrismo es bajo. Tal pareciera que un alto compromiso es un asunto mayormente problemático para el desempeño académico sólo cuando las personas son propensas a enfatizar la importancia de las relaciones sociales y el bienestar de un grupo y, por lo tanto, a ceder en el logro de sus metas personales para no afectar las grupales.

La investigación presenta algunas limitaciones: 1) el tamaño (pequeño) de la muestra, ya que la inferencia estadística (y la generalización teórica) es débil; por ello es importante desarrollar estudios similares en otras universidades para contar con más elementos que permitan avanzar en los resultados presentados. 2) La baja confiabilidad de las escalas para medir las tendencias alocéntricas e idiocéntricas es otro problema; sin embargo, este trabajo representa un avance en el uso de dichas escalas en el idioma español -aunque quizá sea necesario adaptar las preguntas a la cultura mexicana para investigaciones futuras. En cualquier caso, la importancia de este trabajo estriba en sugerir que el co es un factor positivo para las organizaciones, pero que debe administrarse cuidadosamente para no afectar al empleado en otras áreas de su vida. Investigaciones posteriores deberían ahondar en el fenómeno comparando, quizá, diferentes tipos de empleados, en escuelas diferentes (por ejemplo universidades públicas versus universidades privadas) y en culturas que difieren en su nivel de colectivismo e individualismo.

Dicho esto, los resultados presentados tienen implicaciones para los gestores de la educación a nivel posgrado. Por supuesto que en la admisión escolar no se podría discriminar entre aspirantes más o menos comprometidos con su organización y basar las decisiones en este criterio. Pero, lo que quizá podría ayudar a maximizar el promedio escolar es concientizar a los alumnos de que mejorar su aprovechamiento es importante para egresar mejor preparados y, como consecuencia, contribuir mejor a los resultados de su organización y tener mejores oportunidades en ella. Si los estudiantes se convencen de que maximizar sus calificaciones también es de provecho para su organización, se reduce el conflicto de metas (individuales versus grupales) con beneficios para ambas partes. Claro que esto es más crítico en estudiantes cuyas tendencias alocéntricas sean elevadas y las tendencias idiocéntricas sean bajas.

El co es deseable para las organizaciones, aunque podría afectar las calificaciones de los alumnos que trabajan. Este efecto puede variar dependiendo de factores como las tendencias alocéntricas e idiocéntricas. Así, el trabajo y otras esferas de la vida (la escuela en este caso) se contraponen, pues dado que el tiempo, la atención y la energía son limitados, una mayor inversión en un ámbito (el trabajo) puede 
reducir la que se hace en otro (la maestría). Es importante que las escuelas y organizaciones empleadoras traten de reducir el posible conflicto de metas para beneficio de los alumnos empleados y de sus empleadores. Tal vez si el estudiante y la organización se plantean como beneficio mutuo el estudio de un posgrado (para fomentar la innovación, por ejemplo) ambos roles podrían reconciliarse.

\section{Referencias}

Aiken, L. y West, S. (1991). Multiple regression: testing and interpreting interactions. Thousand Oaks, CA: Sage.

Allen, N. y Meyer, J. P. (1990). The measurement and antecedents of affective, continuance and normative commitment to the organization. Journal of Occupational Psychology, 63, 1-18.

doi:10.1111/j.2044-8325.1990.tb00506.x

Alshehri, A. (2017). Student satisfaction and commitment towards a blended learning finance course: A new evidence from using the investment model. Research in International Business and Finance, 41, 423433. doi:10.1016/i.ribaf.2017.04.050

ANUIES (2016). Anuario Estadístico de Educación Superior. Ciclo escolar 2015-2016 (posgrado). Recuperado de http://www.anuies.mx/iinformacion-y-servicios/informacion-estadistica-de-educacionsuperior/anuario-estadistico-de-educacion-superior

Caldwell-Harris, C. y Aycicegi, A. (2006). When personality and culture clash: the psychological distress of allocentrics in an individualist culture and idiocentrics in a collectivist culture. Tanscultural Psychiatry, 43(3), 331-361.

Carrillo, S. y Ríos, J. G. (2013). Trabajo y rendimiento escolar de los estudiantes universitarios. El caso de la Universidad de Guadalajara, México. Revista de la Educación Superior, 42(2), 9-24. Recuperado de http://publicaciones.anuies.mx/pdfs/revista/Revista166 S1A1ES.pdf

Consejo Nacional de Ciencia y Tecnología. (2017). Becas y posgrados. Recuperado de http://www.conacyt.gob.mx/index.php/becas-y-posgrados

Coyle, T. (2015). Relations among general intelligence (g), aptitude test, and GPA: linear effects dominate. Intelligence, 53, 16-22. doi:10.1016/j.intell.2015.08.005

Cropanzano, R. y Mitchell, M. (2005). Social exchange theory: an interdisciplinary review. Journal of Management, 31(6), 874-900. doi:10.1177/0149206305279602

Hair, J., Black, W., Babin, B., Anderson, R. y Tatham, R. (2005). Multivariate data analysis. Upper Saddle River, NJ: Pearson.

Hofstede, G., Hofstede, G. J. y Minkov, M. (2010). Cultures and organizations. Software of the mind. Nueva York: McGrawHill.

Kerlinger, F. y Lee, H. (2006). Foundations of Behavioral Research. Fort Worth, TX: Harcourt College Publishers.

Marsh, H. W. (1991). Employment during high school: character building or a subversion of academic goals? Sociology of Education, 64, 172-189.

Meyer, J. P., Becker, T. y Van Dick, R. (2006). Social identities and commitments at work: toward an integrative model. Journal of Organizational Behavior, 27, 665-683. 
Meyer, J. P., Stanley, D., Herscovitch, L. y Topolnytsky, L. (2001). Affective, continuance, and normative commitment to the organization: a meta-analysis of antecedents, correlates, and consequences. Journal of Vocational Behavior, 61(1), 20-52. doi:10.1006/jvbe.2001.1842

Miller, J. G. (1988). Bridging the content -structure dichotomy: Culture and the self. En M. H. Bond (Ed.), The cross-cultural challenge to social psychology (pp. 266-281). Beverly Hills, CA: Sage.

Mortimer, J., Finch, M., Seongryeol, R., Shanahan, M. y Call, K. (1996). The effects of work intensity on adolescent mental health, achievement, and behavioral adjustment: new evidence from a prospective study. Child Development, 67(3), 1243-1261.

Mowday, R., Porter, L. y Steers, R. (1982). Employee-organization linkages. The psychology of commitment, absenteeism and turnover. Nueva York: Academic Press.

Ng, T. y Feldman, D. (2011). Affective organizational commitment and citizenship behavior: linear and non-linear moderating effects of organizational tenure. Journal of Vocational Behavior, 79, 528-537. doi:10.1016/i.jvb.2011.03.006

Nonis, S. y Hudson, G. (2006). Academic performance of college students: Influence of time spent studying and working. The Journal of Education for Business, 81(3), 151-159. doi:10.3200/JOEB.81.3.151$\underline{159}$

Oettingen, G. (1995). Cross-cultural perspectives on self-efficacy. En A. Bandura (Ed.), Self-efficacy in Changing Societies (149-176). Nueva York: Cambridge University Press.

Ong, A., Phinney, J. y Dennis, J. (2006). Competence under challenge: exploring the protective influence of parental support and ethnic identity in Latino college students. Journal of Adolescence, 29, 961-979. I: doi:10.1016/j.adolescence.2006.04.010

Patton, W. y Smith, E. (2009). Part-time work of high school students and impact on educational outcomes. Australian Journal of Guidance and Counseling, 19(2), 216-224. Recuperado de https://eprints.qut.edu.au/30147/1/c30147.pdf

Rhoades, L. y Eisenberger, R. (2002). Perceived organizational support: a review of the literature. Journal of Applied Psychology, 87(4), 698-714. doi:10.1037/0021-9010.87.4.698

Rochford, C., Connolly, M. y Drennan, J. (2009). Paid part-time employment and academic performance of undergraduate nursing students. Nurse Education Today, 29, 601-606. doi:10.1016/j.nedt.2009.01.004

Singelis, T., Triandis, H. C., Bhawuk, D. y Gelfand, M. (1995). Horizontal and vertical dimensions of individualism and collectivism: a theoretical and measurement refinement. Cross-Cultural Research, 29(3), 240-275. doi:10.1177/106939719502900302

Solinger, O., van Olffen, W. y Roe, R. (2008). Beyond the three-component model of organizational commitment. Journal of Applied Psychology, 93(1), 70-83. doi:10.1037/0021-9010.93.1.70

Triandis, H. C. (2001). Individualism-collectivism and personality. Journal of Personality, 69(6), 907-924. doi: $10.1111 / 1467-6494.696169$

Triandis, H. C. y Gelfand, M. (1998). Converging measurement of horizontal and vertical individualism and collectivism. Journal of Personality and Social Psychology, 74(1), 118-128. doi:10.1037/0022-3514.74.1.118

Triandis, H. C., Leung, K., Villareal, M. J. y Clack, F. I. (1985). Allocentric versus idiocentric tendencies: Convergent and discriminant validation. Journal of Research in Personality, 19(4), 395-415. 
Vedel, A., Thomsen, D. y Larsen, L. (2015). Personality, academic majors and performance: revealing complex patterns. Personality and Individual Differences, 85, 69-76.

Vinarski-Peretz, H., Binyamin, G. y Carmeli, A. (2011). Subjective relational experiences and employee innovative behaviors in the workplace. Journal of Vocational Behavior, 78, 290-304. doi:10.1016/j.paid.2015.04.030

Yamaguchi, S., Kuhlman, D. y Sugimori, S. (1995). Personality correlates of allocentric tendencies in individualist and collectivist cultures. Journal of Cross-Cultural Psychology, 26(6), 658-672. doi: $\underline{10.1177 / 002202219502600609}$ 